MIS, Vol. 7, No. 1, January - June 2008, pp. $1-12$

ISSN 0975-3311

https:/ / doi.org/ 10.12725/ mjs.12.1

\title{
DIGITIZATION OF CONSTANT DEVIATION SPECTROGRAPH
}

\section{Devika Kamath* \& Chandrasekharan K.A.**}

\begin{abstract}
This article is based on the work carried out by the authors as part of the project "Digitization of Physics Experiments with the help of data logger". A computer based measurement requires the conversion of real world analog signal to digital format and transfer of digitized dato into the computer. The objective of this project is to interface a physics experiment to the computer thereby increasing the efficiency and accuracy of the experiment. The experiment selected was the "Analysis of Elemental Spectra using Constant Deviation Spectrograph". The experimental setup was modified and made suitable for the interfacing purpose. It was then interfaced serially to the computer for automatic data acquisition and analysis.
\end{abstract}

\section{Introduction}

A measurement system measures a physical quantity and displays the value on a display device. The basic components of a measurement system are a sensor, signal

* Former MSc student, PG Department of Physics, Christ College, Bangalore - 29, Email: devika13@gmail.com

** Corresponding author, Professor and Head, PG Department of Physics, Christ College, Bangolore - 29, Phone: 08040129339 (O), 08025721984 (R), 9945586502 (M), Email: chandrasekharan.ka@christcollege.edu 
conditioning unit, signal processing unit and the display device. A sensor receives a small amount of energy from the medium and produces an electrical output depending on the physical variable being measured. Signal conditioning and processing methods are employed to transform the sensor output to a form and level necessary to interface with other modules of measurement system.

Advances in computer hardware and software technologies have resulted in easy adoption of the computer in various measurement and control applications. A computer based measurement application requires conversion of real world analog signal into digital format and transfer of the digitized data into the computer. A data acquisition system performs the conversion of analog signal to digital data and digital data to analog signal. Interfacing data acquisition systems to a computer enables the automation of measurement.

Commercially available data acquisition systems are expensive and require custom made software modules for specific applications. The present project aims at designing and implementing a computer interfaced data acquisition system using readily available and less expensive modules for digitization of spectra produced in a constant deviation spectrograph (CDS). The same principle may be employed with minor alterations for data acquisition related to any other physics experiment.

In the conventional method of using a constant deviation spectrograph, the spectrum produced is focused on to a photographic film loaded into the camera. After exposure the film is developed and fixed to obtain the spectral lines on the film. The film is then mounted on to a comparator with a microscope attachment to take distance measurements of the spectral lines. Calibration of CDS setup is done using readings corresponding to standard spectral lines. The measurements are converted to corresponding wavelengths using mathematical equations and using constants derived from calibrations of CDS setup.

In the present project, the spectral lines produced in the CDS are focused on to a photodetector. The small current is amplified and converted to corresponding voltage by Darlington circuit and applied to the input of a digital multimeter. The different lines are brought to the detector by tilting the prism using the rotating drum of the CDS. The rotation of the drum is digitized and converted into a voltage using a ten turn potentiometer attached to the drum. This voltage is applied to the input of another multimeter. The multimeters are provided with memory adapters and are used to interface to the computer through the serial port.

The drum is rotated continuously and the data corresponding to the intensity of light incident on the photodetector and that corresponding to the rotation of the potentiometer at each instant are recorded continuously by the multimeter software. 
The data may be displayed online as a time graph, logging format, or in analog or digital format by the software. The curve fitting and analysis of the logging data is done using Origin software. The wavelengths corresponding to the spectral lines may be found directly from the display.

\section{Experimental Setup}

\section{Constant Deviation Spectrograph}

The spectrograph is an instrument that records on a plate (spectrogram) the spectrum of the radiations under examination. The light after passing through the collimator crosses a prism and is focused to the eyepiece or the camera by the objective of the telescope. The CDS [Fig 2.1] is a spectrograph in which the collimator and telescope are held fixed and the position of the spectral line is varied by rotating the prism. Constant Deviation Spectrograph prism is made with a high refractive index $(1.65$ to 1.70) optical glass which yields excellent performance.

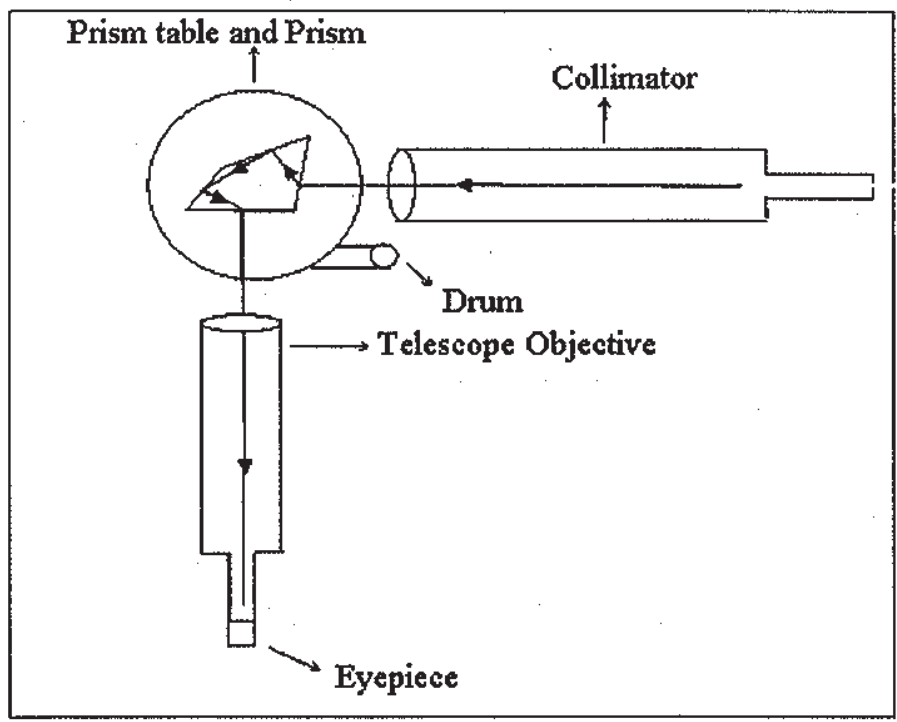

Fig 2.1 : Constant Deviation Spectrograph 


\section{Photodetector and Darlington circuit}

In order to digitize the CDS, the eyepiece is replaced by a photodetector connected to a Darlington circuif [Fig 2.2], which amplifies the current and converts it into voltage, which is applied as the input to the first digital multimeter (channel 7 ). The silicon phototransistor $L 14 \mathrm{Gl}$ which has a narrow reception angle and $V_{C E}$ rating of $5 \mathrm{~V}$ is used as the photodetector. The rotation of the drum is digitized using a 10 turn potentiometer [Fig 2.3] whose output is given to the second digital multimeter (channel 2).

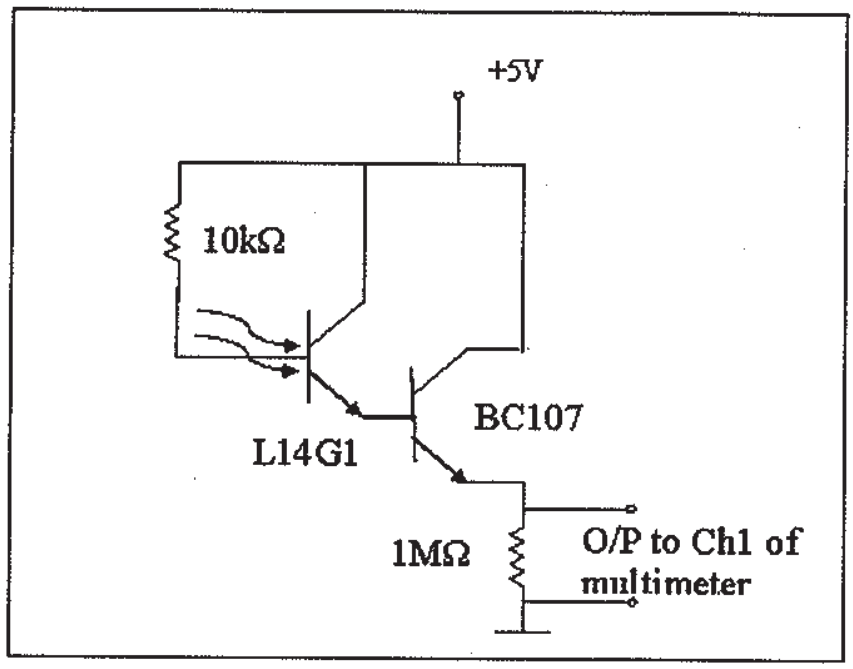

Fig 2.2 : Darlington photodetector circuit

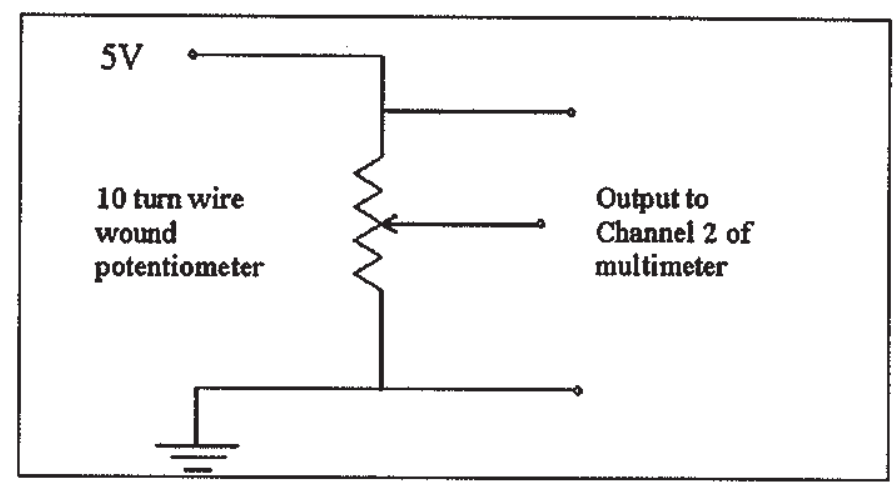

Fig 2.3 : Potentiometer circuit 


\section{Multimeters and Memory adapters}

In this project the interfacing was done using the RISH multimeters and memory adapters [Fig 2.5] which served as an excellent data acquisition system and data logger. The RISH multimeter with its serial RS-232 interface helped in interfacing the experiment to the computer. Two RishMulti 14S digital multimeters were connected to two RishMulti SI232 adapters, via infrared coupling. The outputs from the photodetector Darlington and the potentiometer circuits were fed as inputs to the multimeters (Channel 1 and 2 respectively) [Fig 2.4].

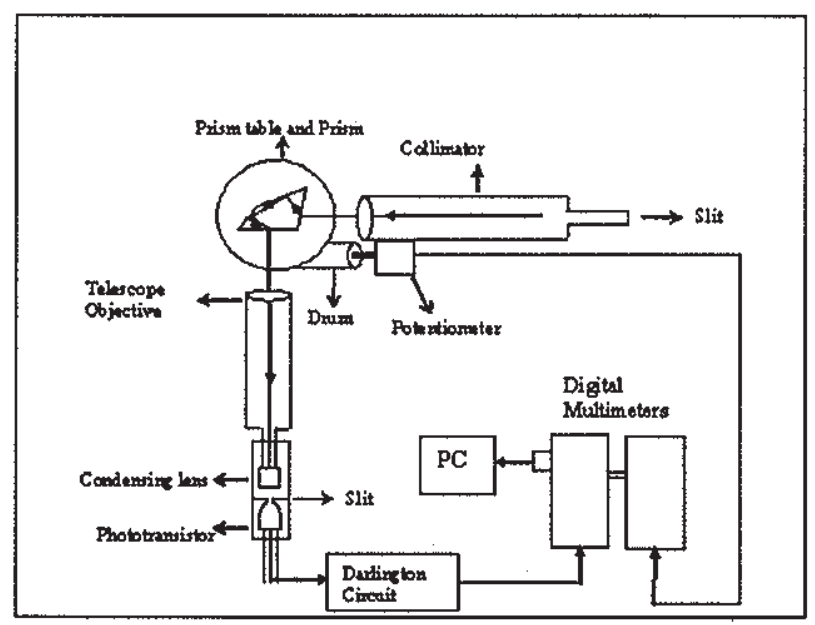

Fig 2.4 : Experimental Setup

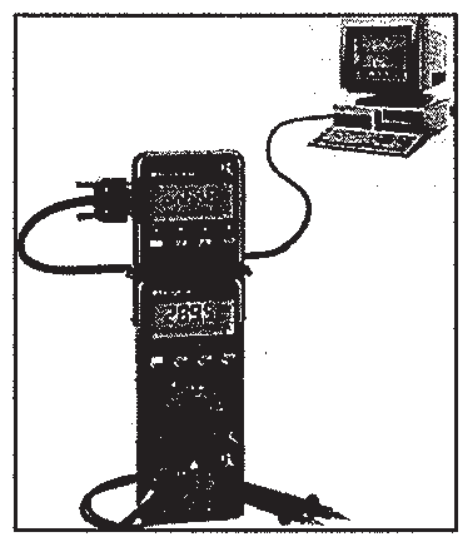

Fig 2.5 : RishMulti 14 S multimeter and RishMulti SI232 Storage Adaptor 
The storage adapter when attached to the hand-held RishMulti multimeter, permits direct transmission of measured data (online) of a single or more Rish Multimeters to the computer. On-site data storage without PC is possible in offline mode of adapter and data can be downloaded to PC afterwards. The data transmission is synchronized by an integrated clock for real time application.

\section{Multimeter Software}

Rishcom 100 software [5] is used for the processing and representation of measurement data on the computer screen. Sampling in the on-line mode can be performed manually with an adjustable sampling interval, or automatically depending upon signal dynamics. The data recorded in the system can be displayed in the following format:

- Data logger

- Multimeter

- $Y(t)$ recorder

- $\quad X-Y$ recorder

\section{Data Collection and Analysis}

\section{Calibration of CDS using spectral lines of mercury}

The CDS was standardized using the mercury source. The analog data from the photodetector and potentiometer circuits are fed to the digital multimeters and analyzed using the Rishcom 100 software. The $Y(t)$ recorder [Fig 3.1] shows two plots which denote the photodetector and potentiometer readings with respect to time. From the plot representing the output of the photodetector, three peaks can be observed corresponding to the standard blue $(4358 \AA)$, green $(5461 \AA)$ and yellow (5770-5790A) lines. The dato logger gives the digitized value of the two outputs which can be used for further analysis. In order to obtain the spectrum as an intensity vs wavelength plot, the potentiometer output is converted to wavelength. The photodetector voltage vs wavelength plot gives the desired spectrum. 


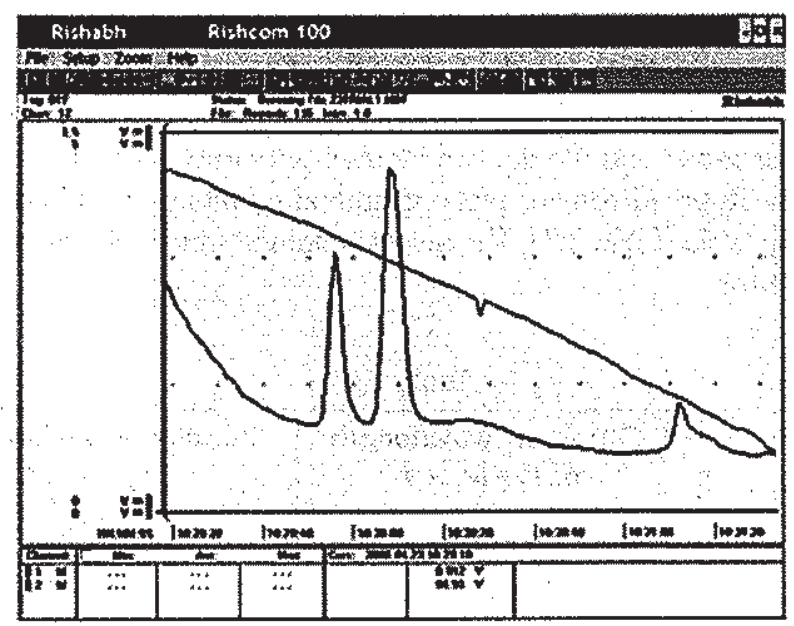

Fig 3.1 : Photodetector and potentiometer voltages as a function of time. (Hg source)

For the analysis of the data, the photodetector and potentiometer outputs from the data logger are copied to Origin 6.0. The value of the potentiometer output corresponding to the three peaks are separately noted and a graph is plotted between the potentiometer voltage vs the standard wavelength of the respective mercury lines: The polynomial curve fitting [Fig 3.2] is done by using the software and the equation for wavelength in terms of potentiometer voltage is obtained.

$Y=4038.85764+28.44835 X+124.29895 X^{2}$

Where, $Y$ is the wavelength $(\AA)$ and $X$ represents the potentiometer voltage $M$.

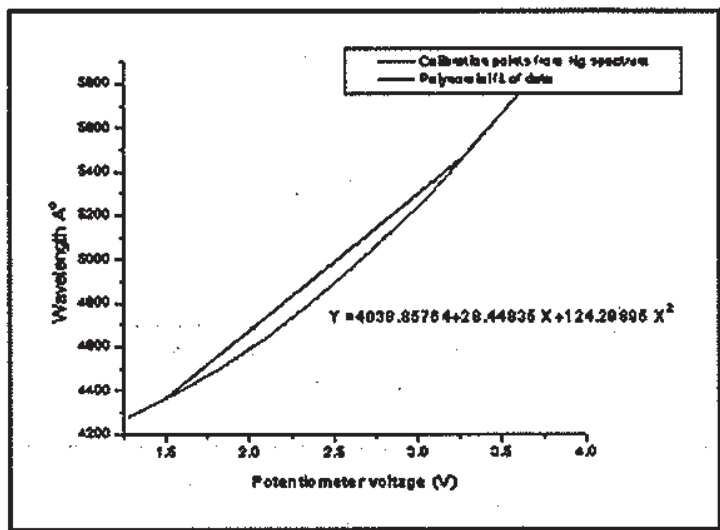

Fig 3.2 : Curve fitting for calibration of CDS 
The wavelengths corresponding to different potentiometer voltages were obtained by using the equation (3.1) with the help of Excel spreadsheet. The graph plotted [Fig 3.3] by taking the photodetector voltage along the $x$-axis and the wavelength along the $y$-axis represents the desired mercury spectrum. The wavelengths of the three peaks are observed from the plot and compared with the standard wavelengths in the following table [Table 3.1]. The results obtained were well within the range of experimental error.

Table 3.1

\begin{tabular}{|l|c|c|}
\hline Color & $\begin{array}{c}\text { Observed wavelength } \\
\text { of } \mathrm{Hg} \text { lines } \AA\end{array}$ & $\begin{array}{c}\text { Standard wavelength of } \\
\text { Hg lines } \AA\end{array}$ \\
\hline Blue & 4357.47 & 4358 \\
Green & 5466.83 & 5461 \\
Yellow & 5776.88 & $5770-5790$ \\
\hline
\end{tabular}

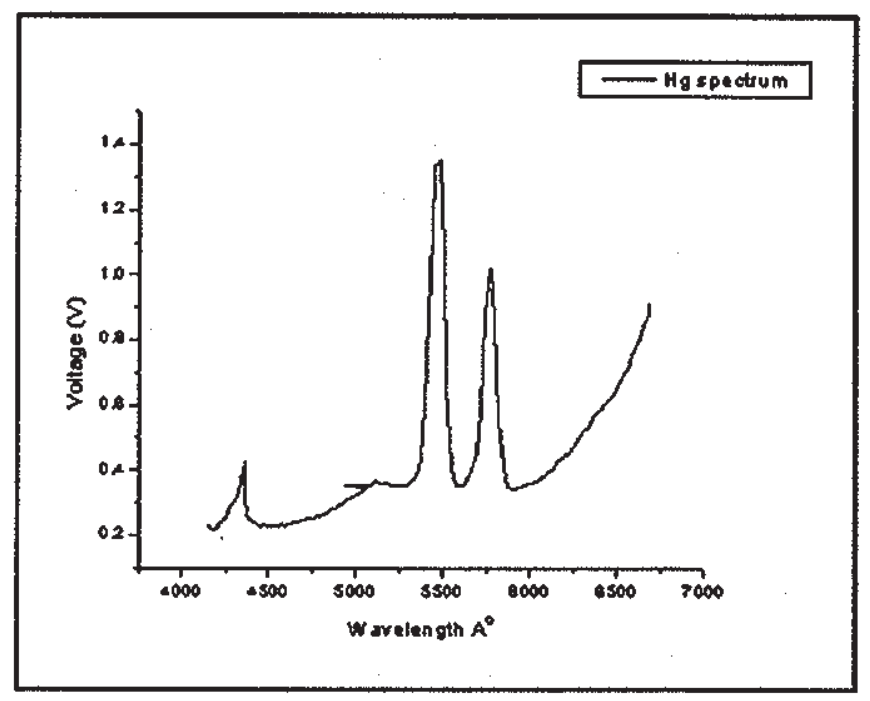

Fig 3.3 : Observed $\mathrm{Hg}$ spectrum 


\section{Analysis of Sodium spectrum}

In order to verify the accuracy of digitization, the spectrum of sodium is studied by using the experimental setup. The $\mathrm{Hg}$ source is replaced by a sodium vapor lamp. The analog data from the CDS is fed into the digital multimeters and analyzed using the Rishcom 100 software. The $y(t)$ recorder gives a plot [Fig 3.4] of the photodetector and potentiometer voltages as a function of time.

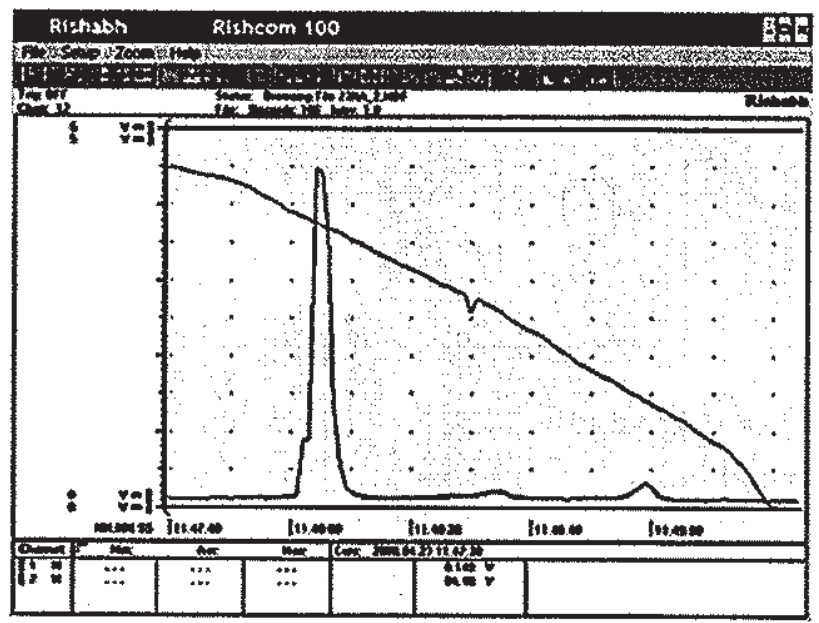

Fig 3.4: Photodetector and potentiometer voltages as a function of time. ( Na source)

The photodetector and potentiometer voltages obtained from the data logger are transferred to Origin 6.0. The potentiometer voltages are converted to wavelengths using the calibration equation (1.1). A plot of the wavelength readings along the $x$-axis and the photodetector voltage along the $y$-axis gives the desired spectrum [Fig 3.5]. The wavelength of the sodium line is observed from the spectrum and compared with the standard wavelength of the sodium line as shown in the following table [Table 3.2].

Table 3.2

\begin{tabular}{|c|c|}
\hline $\begin{array}{c}\text { Observed wavelength of } \\
\text { sodium line } \AA\end{array}$ & $\begin{array}{c}\text { Standard wavelength of } \\
\text { sodium line } \AA\end{array}$ \\
\hline 5879.27 & 5893 \\
\hline
\end{tabular}




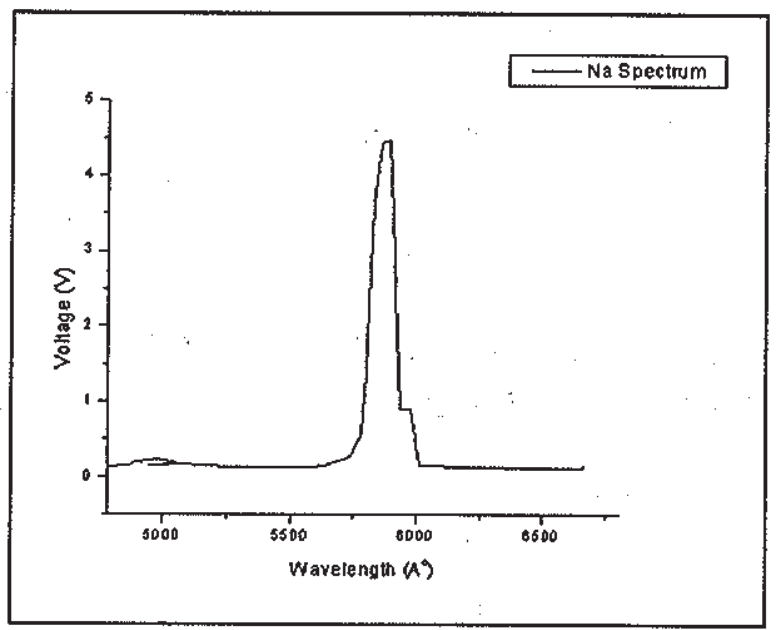

Fig 3.5 : Observed Na spectrum

\section{Analysis of Neon Spectrum}

The neon spectrum is also studied and the wavelength of the neon line is obtained. The sodium vapor lamp is replaced by a neon discharge tube. The analog data from the CDS is fed to the digital multimeters and analyzed using the Rishcom 100 software. The $y(t)$ recorder [Fig 3.6] gives a plot of the photodetector and potentiometer voltages as a function of time.

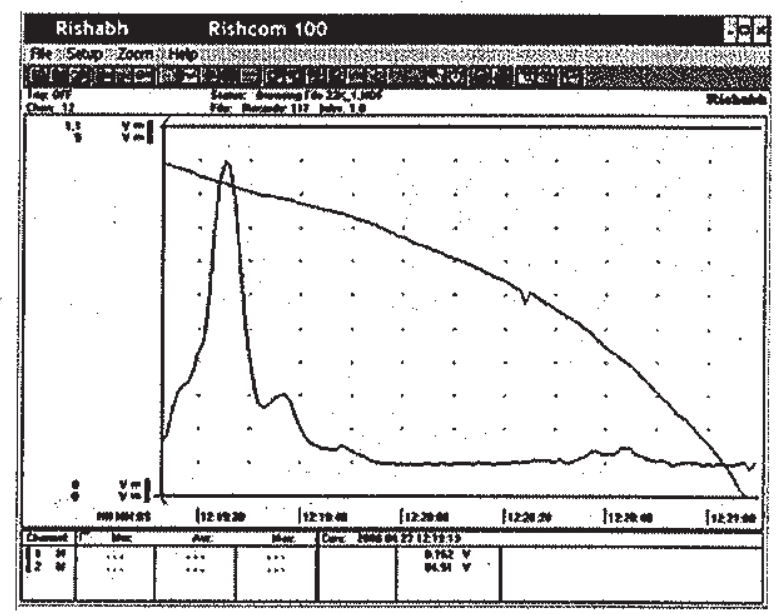

Fig 3.6: Photodetector and potentiometer voltages as a function of time. (Ne spectrum) 
The photodetector and potentiometer voltages obtained from the data Jogger are transferred to Origin 6.0. The potentiometer voltages are converted to wavelengths using the calibration equation (3.1). A plot of the wavelength readings along the $x$-axis and the photodetector voltage along the $y$-axis gives the desired spectrum [Fig 3.7]. The wavelength of the neon line is observed from the spectrum and compared with the standard wavelength of neon as shown in the following table. [Table 3.3].

Table 3.3

\begin{tabular}{|c|c|}
\hline $\begin{array}{c}\text { Observed wavelength of } \\
\text { neon line } \AA\end{array}$ & $\begin{array}{c}\text { Standard wavelength of } \\
\text { neon line } \AA\end{array}$ \\
\hline 6362.79 & 6330 \\
\hline
\end{tabular}

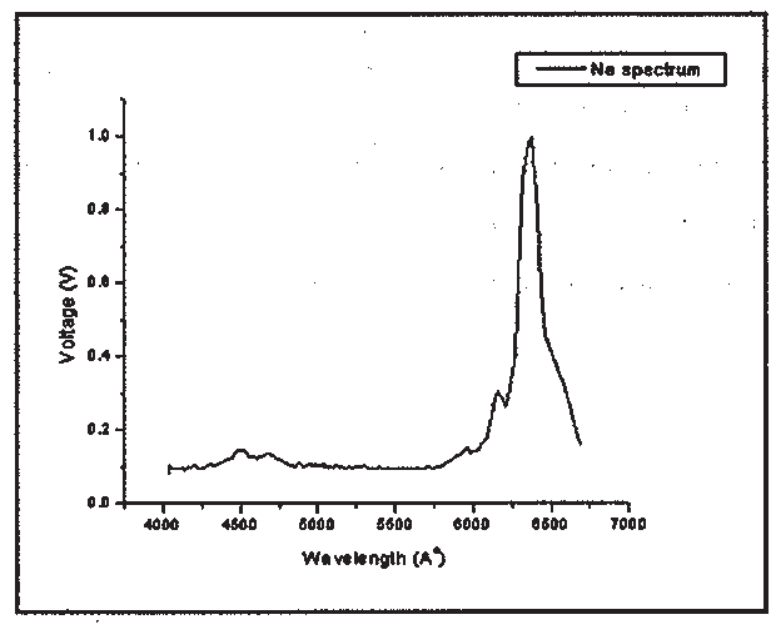

Fig 3.7: Observed Ne spectrum

\section{Conclusion}

The aim of the project was to increase the efficiency, time span, and simplify the procedure for the CDS measurements. The implemented design was found successful in digitizing the CDS to plot the spectra and to read the wavelength of the spectral lines directly on the computer screen. 


\section{Limitations}

Very closely spaced lines remain unresolved. This may be solved by using a finer slit arrangement (adjustable micro slit) in front of the detector.

\section{Suggestions}

In order to completely automate the experiment the drum may be rotated using a stepper motor with a gear system.

Note: The authors thank the Research Development Cell of Christ College, Bangalore for providing financial assistance for undertaking this project.

\section{Bibliography}

1. M.Mathivanan, (2007) PC-Based Instrumentation. Concepts and Practice, Prentice Hall of India Prt.Ltd, new Delhi.

2. Kevin James, (2000) PC-Interfacing and Data Acquisition: Techniques for Measurement, Instrumentation and Control, Newnes.

3. Micheal.H.Tooley, (2005) PC Based instrumentation and control, Newnes.

4. www.fairchildsemi.com/pf/L14G1.html

5. http://www.rishabh.co.in/rishcom_100_software.html 